Homology, Homotopy and Applications, vol.15(1), 2013, pp.127-136

\title{
COHOMOLOGY OF LOOP SPACES OF THE SYMMETRIC SPACE EI
}

\author{
YOUNGGI CHOI
}

\author{
(communicated by J.P.C. Greenlees)
}

\begin{abstract}
We determine the mod $p$ cohomology of the loop space and the double loop space of the symmetric space of exceptional type EI exploiting the Serre spectral sequence and the EilenbergMoore spectral sequence.
\end{abstract}

\section{Introduction}

The 1-connected irreducible symmetric spaces have been classified by E. Cartan. Among them, there are twelve 1-connected compact irreducible symmetric spaces of exceptional type besides of the compact simple Lie group cases. Unlike the classical cases, the cohomology of loop spaces of exceptional types is almost unknown, except for easy cases such as $E I V=E_{6} / F_{4}$ and $F I I=F_{4} / \operatorname{Spin}(9)[\mathbf{4}, \mathbf{5}]$.

In this paper we study the mod $p$ cohomology of the loop space and the double loop space of the symmetric space of exceptional type $E I$. As a homogeneous space, $E I$ is expressed by $E_{6} / P S p(4)$ where $P S p(n)$ is the projective symplectic group. The cohomology of $E I$ is determined in $[\mathbf{7}, \mathbf{8}]$. In fact, the cohomology of $E I$ has two torsion, but it is odd torsion free [8].

This paper is organized as follows: In Section 2, we collect some known facts which will be used in the next section. In Section 3, we calculate the mod 2 cohomology of the loop space of EI exploiting the Serre spectral sequence and the Eilenberg-Moore spectral sequence going to the same destination space. In [2], Bott and Samelson asked whether the cohomology of loop spaces of symmetric spaces has only two torsion. So, in Section 4, we calculate the mod $p$ cohomology of the loop space of $E I$ for odd primes $p$ and prove that the cohomology of the loop space of $E I$ has only two torsion. In Section 5, we apply the results in Sections 4 and 5 to determine the $\bmod p$ (co)homology of the double loop space of $E I$.

\section{Acknowledgements}

I wish to thank Professor M. Mimura for his suggestions and helpful discussions. I also would like to thank the referee for valuable comments.

This work was supported by Basic Science Research Program through the National Research Foundation of Korea (NRF) funded by the Ministry of Education, Science and Technology (2011-0011982). Received January 7, 2012, revised November 24, 2012; published on April 18, 2013. 2000 Mathematics Subject Classification: 55P48, 55R20, 55T10, 57T20.

Key words and phrases: symmetric space $E I$, loop space, Eilenberg-Moore spectral sequence, Serre spectral sequence.

Article available at http://intlpress.com/HHA/v15/n1/a7 and doi:10.4310/HHA.2013.v15.n1.a7

Copyright (C) 2013, International Press. Permission to copy for private use granted. 


\section{Preliminaries}

Let $E(x)$ be the exterior algebra on $x$ and $\Gamma(x)$ the divided power algebra on $x$, which is free over $\gamma_{i}(x)$ as a $\mathbb{F}_{p}$-module with product

$$
\gamma_{i}(x) \gamma_{j}(x)=\left(\begin{array}{c}
i+j \\
j
\end{array}\right) \gamma_{i+j}(x) .
$$

We have homology operations, Dyer-Lashof operations, $Q_{i(p-1)}$ on the $(n+1)$-loop space $\Omega^{n+1} X$

$$
Q_{i(p-1)}: H_{q}\left(\Omega^{n+1} X ; \mathbb{F}_{p}\right) \rightarrow H_{p q+i(p-1)}\left(\Omega^{n+1} X ; \mathbb{F}_{p}\right)
$$

for $0 \leqslant i \leqslant n$ when $p=2$, and for $0 \leqslant i \leqslant n$ and $i+q$ even when $p>2$. They are natural with respect to $(n+1)$-loop maps. In particular, we have $Q_{0} x=x^{p}$. The iterated power $Q_{i}^{a}$ denotes the composition of $Q_{i} a$ times. These operations satisfy the following properties:

Theorem $2.1([6])$. In the path-loop fibration

$$
\Omega^{n+2} X \rightarrow P \Omega^{n+1} X \rightarrow \Omega^{n+1} X,
$$

we have the following:

1. If $x \in H_{*}\left(\Omega^{n+1} X ; \mathbb{F}_{p}\right)$ is transgressive in the Serre spectral sequence, then so is $Q_{i} x$ and $\tau \circ Q_{i(p-1)} x=Q_{(i+1)(p-1)} \circ \tau x$ for each $i, 0 \leqslant i \leqslant n$ where $\tau$ is the transgression.

2. For $p>2$ and $n \geqslant 1$,

$$
d^{2 q(p-1)}\left(x^{p-1} \otimes \tau(x)\right)=-\beta Q_{(p-1)} \tau(x) \text { if } x \in H_{2 q}\left(\Omega^{n+1} X ; \mathbb{F}_{2}\right) .
$$

3. For $p=2, S q_{*}^{1} Q_{i} x=Q_{i-1} x$ if $x \in H_{q}\left(\Omega^{n+1} X ; \mathbb{F}_{2}\right)$ and $q+i$ is even.

We denote the primitives and the indecomposables of $H^{*}\left(X ; \mathbb{F}_{p}\right)$ by $P H^{*}\left(X ; \mathbb{F}_{p}\right)$ and $Q H^{*}\left(X ; \mathbb{F}_{p}\right)$, respectively. In the Eilenberg-Moore spectral sequence associated with the path-loop fibration converging to $H^{*}\left(\Omega X ; \mathbb{F}_{p}\right)$, we have a map

$$
\sigma: Q H^{*}\left(X ; \mathbb{F}_{p}\right) \cong \operatorname{Tor}_{H^{*}\left(X ; \mathbb{F}_{p}\right)}^{-1, *}\left(\mathbb{F}_{p}, \mathbb{F}_{p}\right)=E_{2}^{-1, *} \rightarrow E_{\infty}^{-1, *} \subset H^{*-1}\left(\Omega X ; \mathbb{F}_{p}\right) .
$$

Since the elements of $\operatorname{Tor}_{\mathrm{H}^{*}\left(\mathrm{X} ; \mathbb{F}_{\mathrm{p}}\right)}^{-1, *}\left(\mathbb{F}_{\mathrm{p}}, \mathbb{F}_{\mathrm{p}}\right)$ are primitive and permanent cycles in the Eilenberg-Moore spectral sequence, the above map induces the suspension homomorphism $\sigma: Q H^{*}\left(X ; \mathbb{F}_{p}\right) \rightarrow P H^{*-1}\left(\Omega X ; \mathbb{F}_{p}\right)$.

Theorem $2.2([3])$. Let $X$ be a path connected $H$-space. Then the following is true:

1. The Eilenberg-Moore spectral sequence collapses at $E_{2}$ if and only if $\operatorname{ker} \sigma=0$.

2. The suspension $\sigma: Q H^{\text {odd }}\left(X ; \mathbb{F}_{p}\right) \rightarrow P H^{\text {even }}\left(\Omega X ; \mathbb{F}_{p}\right)$ is injective.

3. The quotient $P H^{\text {even }}\left(\Omega X ; \mathbb{F}_{p}\right) / \sigma\left(Q H^{\text {odd }}\left(X ; \mathbb{F}_{p}\right)\right)$ is obtained by transpotence.

4. The elements in $\operatorname{ker} \sigma$ are dual to elements in the image of the homology transpotence.

Throughout this paper, the subscript of an element means the degree of that element. 


\section{The mod 2 cohomology of the loop space of $E I$}

The space $E I$ is simply connected and $\pi_{2}(E I)=Z_{2}$, so we have the following fibration:

$$
\widetilde{E I} \rightarrow E I \rightarrow K\left(Z_{2}, 2\right),
$$

where $\widetilde{E I}$ is the 2-connected cover of $E I$. Then we have the following morphisms of fibrations:

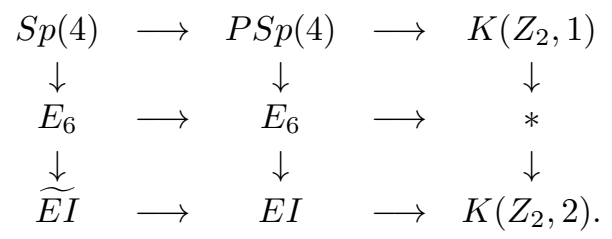

Consider the following fibrations:

$$
\begin{aligned}
& \Omega \widetilde{E I} \longrightarrow * \rightarrow \widetilde{E I} \\
& \Omega E_{6} \longrightarrow \Omega \widetilde{E I} \longrightarrow S p(4) .
\end{aligned}
$$

Recall the following fact from [7]:

Theorem 3.1. $H^{*}\left(\widetilde{E I} ; \mathbb{F}_{2}\right)=E\left(x_{5}, S q^{1} x_{5}, x_{8}, S q^{4} x_{5}, S q^{4} x_{8}, S q^{8} S q^{4} x_{5}\right) \otimes \mathbb{F}_{2}\left[x_{16}\right]$.

First of all, we study the mod 2 cohomology of the loop space of $\widetilde{E I}$.

Proposition 3.2. The mod 2 cohomology of the loop space of $\widetilde{E I}$ is as follows:

$$
H^{*}\left(\Omega \widetilde{E I} ; \mathbb{F}_{2}\right)=\mathbb{F}_{2}\left[y_{2^{i+2}} ; i \geqslant 0\right] /\left(y_{2^{i+2}}^{8}\right) \otimes \Gamma\left(S q^{1} y_{4}, y_{7}, S q^{4} y_{7}\right) \otimes E\left(z_{15}\right) .
$$

Proof. Consider the Eilenberg-Moore spectral sequence of the path-loop fibration (1) converging to $H^{*}\left(\Omega \widetilde{E I} ; \mathbb{F}_{2}\right)$ with

$$
\begin{aligned}
E_{2} & =\operatorname{Tor}_{H^{*}\left(\widetilde{E} I ; \mathbb{F}_{2}\right)}\left(\mathbb{F}_{2}, \mathbb{F}_{2}\right) \\
& =\operatorname{Tor}_{E\left(x_{5}, S q^{1} x_{5}, x_{8}, S q^{4} x_{5}, S q^{4} x_{8}, S q^{8} S q^{4} x_{5}\right) \otimes \mathbb{F}_{2}\left[x_{16}\right]}\left(\mathbb{F}_{2}, \mathbb{F}_{2}\right) \\
& =\Gamma\left(y_{4}, S q^{1} y_{4}, y_{7}, S q^{4} y_{4}, S q^{4} y_{7}, S q^{8} S q^{4} y_{4}\right) \otimes E\left(z_{15}\right) \\
& =\mathbb{F}_{2}\left[y_{2^{i+2}} ; i \geqslant 0\right] /\left(y_{2^{i+2}}^{8}\right) \otimes \Gamma\left(S q^{1} y_{4}, y_{7}, S q^{4} y_{7}\right) \otimes E\left(z_{15}\right) .
\end{aligned}
$$

We also consider the Serre spectral sequence converging to $H^{*}\left(\Omega \widetilde{E I} ; \mathbb{F}_{2}\right)$ for the fibration (2) with

$$
\begin{aligned}
E_{2}= & \mathbb{F}_{2}\left[u_{2}\right] /\left(u_{2}^{16}\right) \otimes\left(\otimes_{i \geqslant 0} \mathbb{F}_{2}\left[\gamma_{2^{i}}\left(u_{8}\right)\right] /\left(\gamma_{2^{i}}\left(u_{8}\right)^{8}\right)\right) \\
& \otimes \Gamma\left(u_{10}, u_{14}, u_{22}\right) \otimes E\left(c_{3}, c_{7}, c_{11}, c_{15}\right),
\end{aligned}
$$

where

$$
H^{*}\left(\Omega E_{6} ; \mathbb{F}_{2}\right)=\mathbb{F}_{2}\left[u_{2}\right] /\left(u_{2}^{16}\right) \otimes\left(\otimes_{i \geqslant 0} \mathbb{F}_{2}\left[\gamma_{2^{i}}\left(u_{8}\right)\right] /\left(\gamma_{2^{i}}\left(u_{8}\right)^{8}\right)\right) \otimes \Gamma\left(u_{10}, u_{14}, u_{22}\right)
$$

in [5] and $H^{*}\left(S p(4) ; \mathbb{F}_{2}\right)=E\left(c_{3}, c_{7}, c_{11}, c_{15}\right)$.

Now we determine all differentials in the above Serre spectral sequence. Since this Serre spectral sequence is a spectral sequence of Hopf algebras, it is enough to 
determine the following transgressions:

$$
\tau\left(u_{2}\right), \tau\left(u_{10}\right), \tau\left(u_{14}\right) .
$$

We have two spectral sequences going to the same destination space $H^{*}\left(\Omega \widetilde{E I} ; \mathbb{F}_{2}\right)$. Comparing these two spectral sequences as a graded vector space, the $E_{2}$-term of the Eilenberg-Moore spectral sequence implies the following transgressions of the Serre spectral sequence:

$$
\begin{aligned}
\tau\left(u_{2}\right) & =c_{3} \\
\tau\left(u_{10}\right) & =0 \\
\tau\left(u_{14}\right) & =0 .
\end{aligned}
$$

If $\tau\left(u_{2}\right)$ were trivial, then $u_{2}$ would survive permanently. But there is no 2-dimensional element in the $E_{2}$-term (3) of the Eilenberg-Moore spectral sequence. So $\tau\left(u_{2}\right)$ should be non-trivial. If $\tau\left(u_{10}\right) \neq 0$, or $\tau\left(u_{14}\right) \neq 0$, then these would imply the following differentials in the Eilenberg-Moore spectral sequence:

$$
\begin{aligned}
d\left(\gamma_{2}\left(S q^{1} y_{4}\right)\right) & =S q^{4} y_{7}, \\
d\left(\gamma_{2}\left(y_{7}\right)\right) & =z_{15},
\end{aligned}
$$

but these are impossible because of the following bidegree reason:

$$
\left|\gamma_{2}\left(S q^{1} y_{4}\right)\right|=(-2,12),\left|S q^{4} y_{7}\right|=(-1,12),\left|\gamma_{2}\left(y_{7}\right)\right|=(-2,16),\left|z_{15}\right|=(-1,16) .
$$

Letting $u_{2}^{2}=u_{4}$ and $c_{3} u_{2}=c_{5}$, we obtain that

$$
\begin{gathered}
E_{\infty}=\mathbb{F}_{2}\left[u_{4}\right] /\left(u_{4}^{8}\right) \otimes\left(\otimes_{i \geqslant 0} \mathbb{F}_{2}\left[\gamma_{2^{i}}\left(u_{8}\right)\right] /\left(\gamma_{2^{i}}\left(u_{8}\right)^{8}\right)\right) \\
\otimes \Gamma\left(u_{10}, u_{14}, u_{22}\right) \otimes E\left(c_{5}, c_{7}, c_{11}, c_{15}\right) .
\end{gathered}
$$

As a graded vector space, the size of this $E_{\infty}$-term is the same as the $E_{2}$-term (3) of the Eilenberg-Moore spectral sequence. This means that the above Eilenberg-Moore spectral sequence collapses at the $E_{2}$-term, and, on the other side, the $E_{\infty}$-term of the Serre spectral sequence has the following extensions:

$$
c_{5}^{2}=u_{10}, \quad c_{7}^{2}=u_{14}, \quad c_{11}^{2}=u_{22} .
$$

So we obtain that

$$
H^{*}\left(\Omega \widetilde{E I} ; \mathbb{F}_{2}\right)=\mathbb{F}_{2}\left[y_{2^{i+2}} ; i \geqslant 0\right] /\left(y_{2^{i+2}}^{8}\right) \otimes \Gamma\left(S q^{1} y_{4}, y_{7}, S q^{4} y_{7}\right) \otimes E\left(z_{15}\right) .
$$

Since the Eilenberg-Moore spectral sequence converging to $H^{*}\left(\Omega \widetilde{E I} ; \mathbb{F}_{2}\right)$ collapses at $E_{2}$, where $E_{2}=\operatorname{Tor}_{H^{*}\left(\widetilde{E} I ; \mathbb{F}_{2}\right)}\left(\mathbb{F}_{2}, \mathbb{F}_{2}\right)$, the Eilenberg-Moore spectral sequence converging to $H_{*}\left(\Omega \widetilde{E I} ; \mathbb{F}_{2}\right)$ also collapses at $E^{2}$, where

$$
\begin{aligned}
E^{2} & =\operatorname{Cotor}^{H_{*}\left(\widetilde{E I} ; \mathbb{F}_{2}\right)}\left(\mathbb{F}_{2}, \mathbb{F}_{2}\right) \\
& \cong \operatorname{Ext}_{H^{*}\left(\widetilde{E I} ; \mathbb{F}_{2}\right)}\left(\mathbb{F}_{2}, \mathbb{F}_{2}\right) \\
& =\mathbb{F}_{2}\left[\nu_{4}, \nu_{5}, \nu_{7}, \nu_{9}, \nu_{11}, \nu_{16}\right] \otimes E\left(\omega_{15}\right) .
\end{aligned}
$$

Hence we get the following:

Corollary 3.3. $H_{*}\left(\Omega \widetilde{E I} ; \mathbb{F}_{2}\right)=\mathbb{F}_{2}\left[\nu_{4}, \nu_{5}, \nu_{7}, \nu_{9}, \nu_{11}, \nu_{16}\right] \otimes E\left(\omega_{15}\right)$. 
For the next step we consider the following fibrations:

$$
\begin{aligned}
& \Omega \widetilde{E I} \longrightarrow \Omega E I \longrightarrow K\left(Z_{2}, 1\right) \\
& \Omega E_{6} \longrightarrow \Omega E I \longrightarrow P S p(4) .
\end{aligned}
$$

Theorem 3.4. The mod 2 cohomology of the loop space of EI is as follows:

$$
H^{*}\left(\Omega E I ; \mathbb{F}_{2}\right)=\mathbb{F}_{2}\left[x_{1}\right] /\left(x_{1}^{16}\right) \otimes \mathbb{F}_{2}\left[y_{2^{i+2}} ; i \geqslant 0\right] /\left(y_{2^{i+2}}^{8}\right) \otimes \Gamma\left(S q^{1} y_{4}, y_{7}, S q^{4} y_{7}\right) .
$$

Proof. Consider two Serre spectral sequences converging to $H^{*}\left(\Omega E I ; \mathbb{F}_{2}\right)$ for the fibration (4) with

$$
E_{2}=\mathbb{F}_{2}\left[y_{2^{i+2}} ; i \geqslant 0\right] /\left(y_{2^{i+2}}^{8}\right) \otimes \Gamma\left(S q^{1} y_{4}, y_{7}, S q^{4} y_{7}\right) \otimes E\left(z_{15}\right) \otimes \mathbb{F}_{2}\left[x_{1}\right],
$$

where $H^{*}\left(K\left(Z_{2}, 1\right) ; \mathbb{F}_{2}\right)=\mathbb{F}_{2}\left[x_{1}\right]$, and for the fibration (5) with

$$
\begin{aligned}
E_{2}= & \mathbb{F}_{2}\left[u_{2}\right] /\left(u_{2}^{16}\right) \otimes\left(\otimes_{i \geqslant 0} \mathbb{F}_{2}\left[\gamma_{2^{i}}\left(u_{8}\right)\right] /\left(\gamma_{2^{i}}\left(u_{8}\right)^{8}\right)\right) \otimes \Gamma\left(u_{10}, u_{14}, u_{22}\right) \\
& \otimes \mathbb{F}_{2}\left[v_{1}\right] /\left(v_{1}^{16}\right) \otimes E\left(b_{3}, b_{7}, b_{11}\right),
\end{aligned}
$$

where $H^{*}\left(P S p(4) ; \mathbb{F}_{2}\right)=\mathbb{F}_{2}\left[v_{1}\right] /\left(v_{1}^{16}\right) \otimes E\left(b_{3}, b_{7}, b_{11}\right)[\mathbf{1}]$.

Since the Serre spectral sequences for the fibration (4) are spectral sequences of Hopf algebras, the source of the first non-trivial differential is an indecomposable element, and its target is a primitive element. Then by dimensional reason, there are only two possible non-trivial differentials as follows: $\tau\left(y_{7}\right)=x_{1}^{8}, \tau\left(z_{15}\right)=x_{1}^{16}$. Now the 7-dimensional generator $b_{7}$ is a permanent cycle because 6-dimensional elements are all decomposables in the Serre spectral sequence for (5). Comparing two Serre spectral sequences for (4) and (5) as graded vector spaces, we can deduce that $\tau\left(y_{7}\right)$ is trivial. However, $\tau\left(z_{15}\right)=x_{1}^{16}$ since there is a truncation of 1-dimensional generator $v_{1}$ in the $E_{2}$-term of the Serre spectral sequences for (5).

So we conclude that the mod 2 cohomology of the loop space of $E I$ is

$$
\mathbb{F}_{2}\left[x_{1}\right] /\left(x_{1}^{16}\right) \otimes \mathbb{F}_{2}\left[y_{2^{i+2}} ; i \geqslant 0\right] /\left(y_{2^{i+2}}^{8}\right) \otimes \Gamma\left(S q^{1} y_{4}, y_{7}, S q^{4} y_{7}\right) .
$$

Corollary 3.5. The cohomology of the loop space of EI has two torsion.

\section{The $\bmod p$ cohomology of the loop space of $E I$}

Recall the following fact from [7]:

Theorem 4.1. For odd primes $p$ we have

$$
H^{*}\left(E I ; \mathbb{F}_{p}\right)=\mathbb{F}_{p}\left[e_{8}\right] /\left(e_{8}^{3}\right) \otimes E\left(e_{9}, e_{17}\right) .
$$

Note that $\beta e_{8}=0$, where $\beta$ is the Bockstein homomorphism because the cohomology of $E I$ is odd torsion free [8].

We also recall the following facts from [5]:

$$
\begin{aligned}
& H^{*}\left(\Omega E_{6} ; \mathbb{F}_{3}\right)=\mathbb{F}_{3}\left[y_{2}\right] /\left(y_{2}^{9}\right) \otimes \Gamma\left(y_{8}, y_{10}, \mathcal{P}^{1} y_{10}, y_{16}, y_{18}, y_{22}\right), \\
& H^{*}\left(\Omega E_{6} ; \mathbb{F}_{p}\right)=\Gamma\left(y_{2}, y_{8}, y_{10}, y_{14}, y_{16}, y_{22}\right), \quad p \geqslant 5 .
\end{aligned}
$$

We will determine the mod $p$ cohomology of the loop space of $E I$ exploiting the following fibrations:

$$
\begin{aligned}
& \Omega E I \longrightarrow * \longrightarrow E I, \\
& \Omega E_{6} \longrightarrow \Omega E I \longrightarrow P S p(4) .
\end{aligned}
$$


Theorem 4.2. For odd primes $p$, the $\bmod p$ cohomology of the loop space of EI is as follows:

$$
H^{*}\left(\Omega E I ; \mathbb{F}_{p}\right)=E\left(x_{7}\right) \otimes \Gamma\left(y_{8}, y_{16}, y_{22}\right) .
$$

Proof. Consider the Eilenberg-Moore spectral sequence for the fibration (6) converging to $H^{*}\left(\Omega E I ; \mathbb{F}_{3}\right)$ with

$$
\begin{aligned}
E_{2} & =\operatorname{Tor}_{H^{*}\left(E I ; \mathbb{F}_{3}\right)}\left(\mathbb{F}_{3}, \mathbb{F}_{3}\right) \\
& =\operatorname{Tor}_{\mathbb{F}_{3}\left[e_{8}\right] /\left(e_{8}^{3}\right) \otimes E\left(e_{9}, e_{17}\right)}\left(\mathbb{F}_{3}, \mathbb{F}_{3}\right) \\
& =E\left(a_{7}\right) \otimes \Gamma\left(a_{8}, a_{16}, a_{22}\right),
\end{aligned}
$$

and also consider the Serre spectral sequence converging to $H^{*}\left(\Omega E I ; \mathbb{F}_{3}\right)$ for the fibration (7) with

$$
E_{2}=E\left(x_{3}, \mathcal{P}^{1} x_{3}, x_{11}, \mathcal{P}^{1} x_{11}\right) \otimes \mathbb{F}_{3}\left[y_{2}\right] /\left(y_{2}^{9}\right) \otimes \Gamma\left(y_{8}, y_{10}, \mathcal{P}^{1} y_{10}, y_{16}, y_{18}, y_{22}\right),
$$

where $H^{*}\left(P S p(4) ; \mathbb{F}_{3}\right)=E\left(x_{3}, \mathcal{P}^{1} x_{3}, x_{11}, \mathcal{P}^{1} x_{11}\right)$.

Comparing these $E_{2}$-terms of two spectral sequences as a graded vector space, we can obtain the following differentials in the Serre spectral sequence:

$$
\begin{aligned}
\tau\left(y_{2}\right) & =x_{3} \\
d_{3}\left(y_{2}^{2}\right) & =x_{3} \otimes y_{2}, \\
\tau\left(y_{2}^{3}\right) & =\mathcal{P}^{1} x_{3} \\
d_{7}\left(y_{2}^{6}\right) & =\mathcal{P}^{1} x_{3} \otimes y_{2}^{3} \\
d_{7}\left(y_{18}\right) & =\mathcal{P}^{1} x_{3} \otimes y_{2}^{6}, \\
\tau\left(y_{10}\right) & =x_{11}, \\
d_{11}\left(\gamma_{i+1}\left(y_{10}\right)\right) & =x_{11} \otimes \gamma_{i}\left(y_{10}\right), i \geqslant 1, \\
\tau\left(\mathcal{P}^{1} y_{10}\right) & =\mathcal{P}^{1} x_{11}, \\
d_{15}\left(\gamma_{i+1}\left(\mathcal{P}^{1} y_{10}\right)\right) & =\mathcal{P}^{1} x_{11} \otimes \gamma_{i}\left(\mathcal{P}^{1} y_{10}\right), i \geqslant 1 .
\end{aligned}
$$

Note that $d_{3}\left(y_{2}^{3}\right)$ cannot be $x_{3} \otimes y_{2}^{2}$ because $d_{3}\left(y_{2}^{3}\right)=3 x_{3} \otimes y_{2}^{2}=0$. Hence $x_{3} \otimes y_{2}^{2}$ is a permanent cycle because there is no element of dimension 8 in the base space $H^{*}\left(P S p(4) ; \mathbb{F}_{3}\right)$. Then we obtain

$$
E_{16}=E\left(x_{3} \otimes y_{2}^{2}\right) \otimes \Gamma\left(y_{8}, y_{16}, y_{22}\right) .
$$

Now by bidegree reason, there are no more non-trivial differentials. Note that $\left|x_{3} \otimes y_{2}^{2}\right|=(3,4),\left|y_{8}\right|=(0,8)$. So $E_{16}=E_{\infty}$. Then letting $x_{3} \otimes y_{2}^{2}=x_{7}$, we get the conclusion for the odd prime 3 .

Next we consider cases of odd primes $p \geqslant 5$. Similarly, we consider the EilenbergMoore spectral sequence of the fibration (6) converging to $H^{*}\left(\Omega E I ; \mathbb{F}_{p}\right)$ with

$$
\begin{aligned}
E_{2} & =\operatorname{Tor}_{H^{*}\left(E I ; \mathbb{F}_{p}\right)}\left(\mathbb{F}_{p}, \mathbb{F}_{p}\right) \\
& =E\left(a_{7}\right) \otimes \Gamma\left(a_{8}, a_{16}, a_{22}\right),
\end{aligned}
$$

and the Serre spectral sequence converging to $H^{*}\left(\Omega E I ; \mathbb{F}_{p}\right)$ for the fibration $(7)$ with

$$
E_{2}=E\left(x_{3}, x_{7}, x_{11}, x_{15}\right) \otimes \Gamma\left(y_{2}, y_{8}, y_{10}, y_{14}, y_{16}, y_{22}\right) \text {. }
$$

Then comparing these $E_{2}$-terms of two spectral sequences as a graded vector space, 
we can obtain the following transgressions in the Serre spectral sequence for (7):

$$
\begin{aligned}
\tau\left(y_{2}\right) & =x_{3}, \\
d_{3}\left(\gamma_{i+1}\left(y_{2}\right)\right) & =x_{3} \otimes \gamma_{i}\left(y_{2}\right), i \geqslant 1, \\
\tau\left(y_{10}\right) & =x_{11}, \\
d_{11}\left(\gamma_{i+1}\left(y_{10}\right)\right) & =x_{11} \otimes \gamma_{i}\left(y_{10}\right), i \geqslant 1, \\
\tau\left(y_{14}\right) & =x_{15}, \\
d_{15}\left(\gamma_{i+1}\left(y_{14}\right)\right) & =x_{15} \otimes \gamma_{i}\left(y_{14}\right), i \geqslant 1 .
\end{aligned}
$$

Hence we get

$$
E_{16}=E\left(x_{7}\right) \otimes \Gamma\left(y_{8}, y_{16}, y_{22}\right) .
$$

Then by bidegree reason, there are no more non-trivial differentials. So $E_{16}=E_{\infty}$, and we get the conclusion for odd primes $p \geqslant 5$.

Corollary 4.3. $H_{*}\left(\Omega E I ; \mathbb{F}_{p}\right)=E\left(\omega_{7}\right) \otimes \mathbb{F}_{p}\left[\nu_{8}, \nu_{16}, \nu_{22}\right]$.

In [2], Bott and Samelson mentioned that they do not know whether the cohomology of the loop space of a symmetric space has only two torsion. Now we determine whether the cohomology of the loop space of EI has only two torsion. Rationally we have

$$
\begin{aligned}
H^{*}\left(\Omega E_{6} ; \mathbb{Q}\right) & =\mathbb{Q}\left[y_{2}, y_{8}, y_{10}, y_{14}, y_{16}, y_{22}\right], \\
H^{*}(P S p(4) ; \mathbb{Q}) & =E\left(x_{3}, x_{7}, x_{11}, x_{15}\right) .
\end{aligned}
$$

Then, similar to the mod $p$ case, comparing the Eilenberg-Moore spectral sequence for the fibration $(6)$ converging to $H^{*}(\Omega E I ; \mathbb{Q})$ and the Serre spectral sequence converging to $H^{*}(\Omega E I ; \mathbb{Q})$ for the fibration $(7)$, we can determine the rational cohomology of the loop space of $E I$ as follows:

$$
H^{*}(\Omega E I ; \mathbb{Q})=E\left(x_{7}\right) \otimes \mathbb{Q}\left[y_{8}, y_{16}, y_{22}\right] .
$$

Note that for a space $X$

$$
\begin{aligned}
\operatorname{dim}_{\mathbb{F}_{p}}\left(H^{*}\left(X ; \mathbb{F}_{p}\right)\right) & \geqslant \operatorname{dim} \mathbb{F}_{p}\left(H^{*}(X ; Z) / \text { torsion } \otimes \mathbb{F}_{p}\right) \\
& =\operatorname{dim}_{\mathbb{Q}}\left(H^{*}(X ; \mathbb{Q})\right) .
\end{aligned}
$$

So if $\operatorname{dim}_{\mathbb{F}_{p}}\left(H^{*}\left(X ; \mathbb{F}_{p}\right)\right)=\operatorname{dim}_{\mathbb{Q}}\left(H^{*}(X ; \mathbb{Q})\right)$, then the Bockstein spectral sequence converging to $\left(H^{*}(X ; Z) /\right.$ torsion $) \otimes \mathbb{F}_{p}$ collapses at $E_{1}$ where $E_{1}=H^{*}\left(X ; \mathbb{F}_{p}\right)$. This implies that $H^{*}(X ; Z)$ is $p$-torsion free.

Since $\operatorname{dim}_{\mathbb{F}_{p}}\left(H^{*}\left(\Omega E I ; \mathbb{F}_{p}\right)\right)=\operatorname{dim}_{\mathbb{Q}}\left(H^{*}(\Omega E I ; \mathbb{Q})\right)$, the cohomology of the loop space of $E I$ is odd torsion free. Hence with Corollary 3.5, we get the following conclusion:

Corollary 4.4. The cohomology of the loop space of EI has only two torsion.

\section{The (co)homology of the double loop space of $E I$}

We determine the mod $p$ (co)homology of the double loop space of $E I$ by applying the results in Sections 4 and 5 . Since $\pi_{2}(E I)=Z_{2}, \pi_{0}\left(\Omega^{2} E I\right)=Z_{2}$. Let $\Omega_{0}^{2} E I$ be the 
zero component of $\Omega^{2} E I$. By looping the fibration twice, $\widetilde{E I} \rightarrow E I \rightarrow K\left(Z_{2}, 2\right)$, we get $\Omega_{0}^{2} E I \simeq \Omega^{2} \widetilde{E I}$. Now we compute $H^{*}\left(\Omega^{2} \widetilde{E I} ; \mathbb{F}_{2}\right)$ instead of $H^{*}\left(\Omega_{0}^{2} E I ; \mathbb{F}_{2}\right)$.

Lemma 5.1. The cohomology suspension map

$$
\sigma: Q H^{*}\left(\Omega \widetilde{E I} ; \mathbb{F}_{2}\right) \rightarrow P H^{*-1}\left(\Omega^{2} \widetilde{E I} ; \mathbb{F}_{2}\right)
$$

is injective.

Proof. From Theorem 2.2, if $x \in \operatorname{ker} \sigma$ for $x \in Q H^{*}\left(\Omega \widetilde{E I} ; \mathbb{F}_{2}\right)$, the degree of $x$ should be of the form $n \times 2^{k}+2$ for $n \geqslant 1$ and $k \geqslant 2$. There are $y_{2^{i+2}}, i \geqslant 0, \gamma_{2^{i}}\left(S q^{1} y_{4}\right)$, $i \geqslant 1, \gamma_{2^{i}}\left(y_{7}\right), i \geqslant 1, \gamma_{2^{i}}\left(S q^{1} y_{7}\right), i \geqslant 1$ in $Q H^{\text {even }}\left(\Omega \widetilde{E I} ; \mathbb{F}_{2}\right)$, but degrees of these elements cannot be of the form $n \times 2^{k}+2$ for $k \geqslant 2$. Hence the suspension

$$
\sigma: Q H^{*}\left(\Omega \widetilde{E I} ; \mathbb{F}_{2}\right) P H^{*-1}\left(\Omega^{2} \widetilde{E I} ; \mathbb{F}_{2}\right)
$$

is injective.

By Theorem 2.2 we obtain the following corollary:

Corollary 5.2. The Eilenberg-Moore spectral sequence converging to $H^{*}\left(\Omega^{2} \widetilde{E I} ; \mathbb{F}_{2}\right)$ collapses at $E_{2}$, where

$$
E_{2}=\operatorname{Tor}_{H^{*}\left(\Omega \widetilde{E I} ; \mathbb{F}_{2}\right)}\left(\mathbb{F}_{2}, \mathbb{F}_{2}\right) .
$$

Corollary 5.3. The Eilenberg-Moore spectral sequence converging to $H_{*}\left(\Omega^{2} \widetilde{E I} ; \mathbb{F}_{2}\right)$ collapses at $E^{2}$, where

$$
E^{2}=\operatorname{Ext}_{H^{*}\left(\Omega \widetilde{E I} ; \mathbb{F}_{2}\right)}\left(\mathbb{F}_{2}, \mathbb{F}_{2}\right) \cong \operatorname{Cotor}^{H_{*}\left(\Omega \widetilde{E I} ; \mathbb{F}_{2}\right)}\left(\mathbb{F}_{2}, \mathbb{F}_{2}\right) .
$$

Theorem 5.4. The mod 2 homology of the double loop space of EI is as follows:

$$
\begin{aligned}
H_{*}\left(\Omega_{0}^{2} E I ; \mathbb{F}_{2}\right)= & E\left(Q_{1}^{a} z_{3}: a \geqslant 0\right) \otimes \mathbb{F}_{2}\left[\beta_{3} Q_{1}^{a+3} z_{3}: a \geqslant 0\right] \otimes \mathbb{F}_{2}\left[w_{14}\right] \\
& \otimes \mathbb{F}_{2}\left[Q_{1}^{a} z_{4}: a \geqslant 0\right] \otimes \mathbb{F}_{2}\left[Q_{1}^{a} z_{6}: a \geqslant 0\right] \otimes \mathbb{F}_{2}\left[Q_{1}^{a} z_{10}: a \geqslant 0\right],
\end{aligned}
$$

where $\beta_{3}$ is the tertiary homology Bockstein operator.

Proof. Consider the Eilenberg-Moore spectral sequence converging to $H_{*}\left(\Omega^{2} \widetilde{E I} ; \mathbb{F}_{2}\right)$ with

$$
E^{2}=\operatorname{Ext}_{H^{*}\left(\Omega \widetilde{E I} ; \mathbb{F}_{2}\right)}\left(\mathbb{F}_{2}, \mathbb{F}_{2}\right)=\operatorname{Cotor}^{H_{*}\left(\Omega \widetilde{E I} ; \mathbb{F}_{2}\right)}\left(\mathbb{F}_{2}, \mathbb{F}_{2}\right) .
$$

Then by Theorem 2.1, Corollary 3.3, and Corollary 5.3,

$$
\begin{aligned}
E^{\infty}= & E^{2} \\
= & E\left(Q_{1}^{a} z_{3}: a \geqslant 0\right) \otimes \mathbb{F}_{2}\left[w_{2^{i+5}-2}: i \geqslant 0\right] \otimes \mathbb{F}_{2}\left[w_{14}\right] \\
& \otimes \mathbb{F}_{2}\left[Q_{1}^{a} z_{4}: a \geqslant 0\right] \otimes \mathbb{F}_{2}\left[Q_{1}^{a} z_{6}: a \geqslant 0\right] \otimes \mathbb{F}_{2}\left[Q_{1}^{a} z_{10}: a \geqslant 0\right] .
\end{aligned}
$$

Consider the Serre spectral sequence associated with the fibration

$$
\Omega^{2} E_{6} \rightarrow \Omega^{2} \widetilde{E I} \rightarrow \Omega S p(4) .
$$

It is easy to compute that $H_{*}\left(\Omega S p(4) ; \mathbb{F}_{2}\right)=\mathbb{F}_{2}\left[a_{2}, a_{6}, a_{10}, a_{14}\right]$. 
Recall the following in [5]:

$$
\begin{aligned}
H_{*}\left(\Omega^{2} E_{6} ; \mathbb{F}_{2}\right)= & E\left(z_{1}\right) \otimes E\left(Q_{1}^{a} z_{7}: a \geqslant 0\right) \otimes \mathbb{F}_{2}\left[\beta_{3} Q_{1}^{a+2} z_{7}: a \geqslant 0\right] \\
& \otimes \mathbb{F}_{2}\left[Q_{1}^{a} z_{9}: a \geqslant 0\right] \otimes \mathbb{F}_{2}\left[Q_{1}^{a} z_{13}: a \geqslant 0\right] \otimes \mathbb{F}_{2}\left[Q_{1}^{a} z_{21}: a \geqslant 0\right] .
\end{aligned}
$$

As a graded vector space, the $E^{\infty}$-term of the Serre spectral sequence should have the same size in every total degree as the above $E^{\infty}$-term of the Eilenberg-Moore spectral sequence. Then we obtain the following transgression: $\tau\left(a_{2}\right)=z_{1}$, and $a_{2} \otimes z_{1}$ survives permanently. Let $a_{2} \otimes z_{1}=z_{3}$. Then by (8) and (9),

$$
\begin{aligned}
H_{*}\left(\Omega^{2} \widetilde{E I} ; \mathbb{F}_{2}\right)= & E\left(Q_{1}^{a} z_{3}: a \geqslant 0\right) \otimes \mathbb{F}_{2}\left[\beta_{3} Q_{1}^{a+3} z_{3}: a \geqslant 0\right] \otimes \mathbb{F}_{2}\left[w_{14}\right] \\
& \otimes \mathbb{F}_{2}\left[Q_{1}^{a} z_{4}: a \geqslant 0\right] \otimes \mathbb{F}_{2}\left[Q_{1}^{a} z_{6}: a \geqslant 0\right] \otimes \mathbb{F}_{2}\left[Q_{1}^{a} z_{10}: a \geqslant 0\right] .
\end{aligned}
$$

Corollary 5.5. The mod 2 cohomology of the double loop space of EI is as follows:

$$
\begin{aligned}
H^{*}\left(\Omega_{0}^{2} E I ; \mathbb{F}_{2}\right)= & E\left(e_{3}, e_{7}, e_{15}\right) \otimes E\left(\beta^{3} \alpha_{2^{i+5}-2}: i \geqslant 0\right) \otimes \Gamma\left(\alpha_{2^{i+5}-2}: i \geqslant 0\right) \otimes \Gamma\left(\alpha_{14}\right) \\
& \otimes \Gamma\left(e_{2^{i} \times 5-1}: i \geqslant 0\right) \otimes \Gamma\left(e_{2^{i} \times 7-1}: i \geqslant 0\right) \otimes \Gamma\left(e_{2^{i} \times 11-1}: i \geqslant 0\right),
\end{aligned}
$$

where $\beta^{3}$ is the tertiary Bockstein operator.

For odd prime cases, we recall the following in $[\mathbf{9}]$ :

Theorem 5.6. Let $X$ be a 1-connected $H$-space of finite type and let $x \in \operatorname{Ker} \sigma$. Assume $p$ is an odd prime. Then either there is an indecomposable class $u \in H^{2 m+1}(x)$ such that $\beta_{k} \mathcal{P}^{I}$ for $I=\left(p^{k-l} m, \ldots, m\right)$ is defined and contains $x$, or else there is an indecomposable class $v \in H^{2 s}(x)$ of height $p^{r}$ such that $\beta_{k} \mathcal{P}^{J} \psi_{r}(v)$ for $J=$ $\left.\left(p^{k-l}\left(p^{r} s-1\right), \ldots, p\left(p^{r} s-1\right)\right)\right)$ is defined and contains $x$.

Then we get the following corollary:

Corollary 5.7. Let $X$ be a 1-connected $H$-space of finite type and $p$ be an odd prime. Then if $H^{*}(X ; Z)$ is odd torsion free, the Eilenberg-Moore spectral sequence converging to $H^{*}\left(\Omega X ; \mathbb{F}_{p}\right)$ collapses at $E_{2}$, where

$$
E_{2}=\operatorname{Tor}_{H^{*}\left(X ; \mathbb{F}_{p}\right)}\left(\mathbb{F}_{p}, \mathbb{F}_{p}\right) .
$$

Localized at $p$ for any odd primes, $E I$ is 7-connected. Since the cohomology of the loop space of $E I$ is odd torsion free by Corollary 4.4, we get the following:

Corollary 5.8. The Eilenberg-Moore spectral sequence converging to $H^{*}\left(\Omega_{0}^{2} E I ; \mathbb{F}_{p}\right)$ collapses at $E_{2}$, where

$$
E_{2}=\operatorname{Tor}_{H^{*}\left(\Omega E I ; \mathbb{F}_{p}\right)}\left(\mathbb{F}_{p}, \mathbb{F}_{p}\right) .
$$

Corollary 5.9. The Eilenberg-Moore spectral sequence converging to $H_{*}\left(\Omega_{0}^{2} E I ; \mathbb{F}_{p}\right)$ collapses at $E^{2}$, where

$$
E^{2}=\operatorname{Ext}_{H^{*}\left(\Omega E I ; \mathbb{F}_{p}\right)}\left(\mathbb{F}_{p}, \mathbb{F}_{p}\right) \cong \operatorname{Cotor}^{H_{*}\left(\Omega E I ; \mathbb{F}_{p}\right)}\left(\mathbb{F}_{p}, \mathbb{F}_{p}\right) .
$$

Theorem 5.10. For odd primes $p$, the mod $p$ homology of the double loop space of $E I$ is as follows:

$$
\begin{aligned}
H_{*}\left(\Omega_{0}^{2} E I ; \mathbb{F}_{p}\right)= & \mathbb{F}_{p}\left[z_{6}\right] \otimes E\left(Q_{(p-1)}^{a} z_{7}: a \geqslant 0\right) \otimes \mathbb{F}_{p}\left[\beta Q_{(p-1)}^{a+1} z_{7}: a \geqslant 0\right] \\
& \otimes E\left(Q_{(p-1)}^{a} z_{15}: a \geqslant 0\right) \otimes \mathbb{F}_{p}\left[\beta Q_{(p-1)}^{a+1} z_{15}: a \geqslant 0\right] \\
& \otimes E\left(Q_{(p-1)}^{a} z_{21}: a \geqslant 0\right) \otimes \mathbb{F}_{p}\left[\beta Q_{(p-1)}^{a+1} z_{21}: a \geqslant 0\right] .
\end{aligned}
$$


Proof. Consider the Eilenberg-Moore spectral sequence converging to $H_{*}\left(\Omega_{0}^{2} E I ; \mathbb{F}_{p}\right)$ with

$$
E^{2}=\operatorname{Ext}_{H^{*}\left(\Omega E I ; \mathbb{F}_{p}\right)}\left(\mathbb{F}_{p}, \mathbb{F}_{p}\right)=\operatorname{Cotor}^{H_{*}\left(\Omega E I ; \mathbb{F}_{p}\right)}\left(\mathbb{F}_{p}, \mathbb{F}_{p}\right) .
$$

Then by Theorem 2.1, Corollary 4.3, and Corollary 5.9,

$$
\begin{aligned}
E^{\infty}= & E^{2} \\
= & \mathbb{F}_{p}\left[z_{6}\right] \otimes E\left(Q_{(p-1)}^{a} z_{7}: a \geqslant 0\right) \otimes \mathbb{F}_{p}\left[\beta Q_{(p-1)}^{a+1} z_{7}: a \geqslant 0\right] \\
& \otimes E\left(Q_{(p-1)}^{a} z_{15}: a \geqslant 0\right) \otimes \mathbb{F}_{p}\left[\beta Q_{(p-1)}^{a+1} z_{15}: a \geqslant 0\right] \\
& \otimes E\left(Q_{(p-1)}^{a} z_{21}: a \geqslant 0\right) \otimes \mathbb{F}_{p}\left[\beta Q_{(p-1)}^{a+1} z_{21}: a \geqslant 0\right] .
\end{aligned}
$$

Corollary 5.11. For odd primes $p$, the mod $p$ cohomology of the double loop space of $E I$ is as follows:

$$
\begin{aligned}
H^{*}\left(\Omega_{0}^{2} E I ; \mathbb{F}_{p}\right)= & \Gamma\left(\alpha_{6}\right) \otimes E\left(e_{7}, e_{15}, e_{21}\right) \otimes E\left(\beta \alpha_{8 p^{i+1}-2}: i \geqslant 0\right) \otimes \Gamma\left(\alpha_{8 p^{i+1}-2}: i \geqslant 0\right) \\
& \otimes E\left(\beta \alpha_{16 p^{i+1}-2}: i \geqslant 0\right) \otimes \Gamma\left(\alpha_{16 p^{i+1}-2}: i \geqslant 0\right) \\
& \otimes E\left(\beta \alpha_{22 p^{i+1}-2}: i \geqslant 0\right) \otimes \Gamma\left(\alpha_{22 p^{i+1}-2}: i \geqslant 0\right) .
\end{aligned}
$$

\section{References}

[1] P. Baum and W. Browder, The cohomology of quotients of classical groups, Topology 3 (1965), no. 4, 305-336.

[2] R. Bott and H. Samelson, Applications of the theory of Morse to symmetric spaces, Amer. J. Math. 80 (1958), no. 4, 964-1029.

[3] W. Browder, On differential Hopf algebras, Trans. Amer. Math. Soc. 107 (1963), no. 1, 153-176.

[4] Y. Choi and S. Yoon, Homology of the triple loop space of the exceptional Lie group $F_{4}$, J. Korean Math. Soc. 35 (1998), no. 1, 149-164.

[5] Y. Choi and S. Yoon, Homology of the double and the triple loop space of the exceptional Lie group $E_{6}, E_{7}$, and $E_{8}$, Manuscripta Math. 103 (2000), no. 1, 101-116.

[6] F.R. Cohen, T. Lada and J.P. May, The homology of iterated loop spaces, Lecture Notes Math. 533, Springer-Verlag, New York, 1976.

[7] K. Ishitoya, Cohomology of the symmetric space EI, Proc. Japan Acad. Ser. A Math. Sci. 53 (1977), no. 2, 56-60.

[8] K. Ishitoya and H. Toda, On the cohomology of irreducible symmetric spaces of exceptional type, J. Math. Kyoto Univ. 17 (1977), no. 2, 225-243.

[9] D. Kraines, The kernel of the loop suspension map, Illinois J. Math. 21 (1977), no. 1, 91-108.

Younggi Choi yochoi@snu.ac.kr

Department of Mathematics Education, Seoul National University, Seoul 151-748, Korea 